body-size are all, unexceptionally, the most definitely nocturnal in their habits, while those of larger size are almost entirely diurnal. This in itself is an interesting association. It would also appear that the small nocturnal lemurs have larger eyeballs than the larger diurnal lemurs. Finally, the small nocturnal lemurs live either in pairs or in single families, while the larger diurnal lemurs tend to live in small groups which contain the members of at least one other family.

Since the lemurs represent the most primitive, as well as the oldest, type of Primates, being, indeed, best regarded as a relict group, it may reasonably be assumed that they preserve the social habits of even earlier ancestral Primates. The point is not very important, but it is worth mention because it is known that the earliest Primates were not much larger than small rats, and from this-together with the evidence cited in this communication-it may be inferred that they were probably nocturnal in their habits, and lived in pairs or single families, as do the closely related primatoid menotyphlous insectivores (for example, Tupaiidæ) to this day.

The evidence provided by the lemurs suggests that small arboreal Primates are better able to survive when they are active at night than they are when they are active during the day. Large eyes provide them with the necessary visual power to pursue their nocturnal activities, and these activities can most effectively be pursued in a solitary manner or in pairs or in small family groups. These seem to be the facts for the lemurs.

The reverse conditions seem to be equally closely connected. Now, what are the facts for the remaining suborders and families of Primates? Are these facts or is this association of conditions corroborated by the conditions existing in any other group of Primates? It may be said at once that the evidence is strikingly corroborative, and where it is exceptional, very enlightening.

The exception to the rule of having all four variables positively correlated is provided by a single family among the Platyrrhini or Old World monkeys, the Hapalidæ, consisting of the marmosets and tamarins. These animals are about the size of a small squirrel, but they all have small or medium-sized eyes, are diurnal in their habits, and are said to live in pairs or in single families. The two important correlations are here ( 1 ) between body size and the habit of living in pairs or in single families, and (2), between diurnal activities and small or medium-sized eyes. I shall return to a discussion of the significance of these facts presently. Meanwhile, we must be grateful to Nature for providing us with exceptional cases, for these often serve to throw light on the relative importance of variables which are otherwise too closely correlated to lend themselves to the necessary separation and analysis.

Finally, and most significantly, among the ten genera of the family Cebidæ (South American monkeys) embracing some ninety-three different species, the species of smallest size (embracing ten species) all belong to the same genus Aotes, the douroucoulis or night-monkeys. These are the only monkeys, among the Cebidæ, which have large eyes, are nocturnal in their habits, and live in pairs or in single families. They are about the size of a squirrel.

All other Primates of the platyrrhine and catarrhine series are of relatively large body-size, with small or medium-sized eyes, are diurnal in their activities, and live in communities or bands. (By a community or band is meant a group consisting of the members of two or more families.)

We have here, then, an almost perfect correlation between (1) body-size, (2) kind of primary social group, (3) waking or sleeping activity habits, and (4) size of eyes.

Small Primates are likely to have large eyes, to be nocturnal, and to live either in solitude, in pairs, or in small family groups. Large Primates are likely to have small or medium-sized eyes, to be diurnal, and to live in bands or communities.

The conditions existing among the marmosets indicate that when small Primates are diurnal their eyes are small or of medium size, and that they retain their habit of living in pairs or in single families.

It need scarcely be pointed out here that even a complete positive correlation does not necessarily imply a causal relationship between the variables found in association. Such an association may be due to still other conditions, and it is a safe general rule to assume that this is the case until erery effort has been exhausted to prove it so. In the present case, in virtue of the nature of the data, such attempts to prove the existence of significant additional conditions are not readily feasikle. Our task must therefore lie in an attempt to educe the order of most probable relationship existing ketween the four variables we have noted. In this we are greatly assisted by the apparently aberrant conditions found among the marmosets. From the latter it appears that body size is directly associated with small social groups limited to a single pair or a family, while size of eyes is most directly associated, as would be expected, with type of waking activ ity.

To conclude, then, the evidence suggests that increase in body size (and, presumably, weight) may have been a potent factor in releasing the early Anthro. poidea from their crepuscular halits, $\mathrm{k} y$ enabling them to hold their own against most aggressors, and that this together with the potentialities released by the assumption of a diurnal life led to the development of more extended social relations with animals of their orn species. It is perhaps worth pointing out that this is not a speculation, but an interpretation of facts to which a high degree of probability is attached.

A fuller account of these tindings will be published in the American Anthropologist.

\section{ADVERTISEMENT OF PROPRIETARY MEDICINES}

"M ASS OBSERVATION" has issued the results of their investigation among their national panel of voluntary informants of he attitude of these informants to patent medicines. These informants do not represent a typical cross-section of the population, but the majority of them are dissatisfied with the present position and ask for further Government control of the price, sale and advertisement of such products, because they make ex ggerated claims, play upon the fears of illness and prevent people from going to a medical man, so that illnesses which might be recognized early, and arrested, become chronic. These informants do not, on the other hand, wish to see medically approved remedies removed from the market.

Emphasis is given to this report by an annotation in the British Medical Journal (Sept. 25 , .943, p. 398) which discusses the experiments performed by Ivy, 
Roback and Stein (Quart. Bull. Northwest Univ. Med. School, 16, 298; 1942) with 'Carter's Little Liver Pills'. Using dogs, these workers injected the pills in solution in alcohol intravenously, or in solution in water intraduodenally, giving the equivalent of 2-4 pills per dose. None of the doses produced any significant increase in the flow of bile or of the pressure inside the gall bladder. The makers of 'Carter's Little Liver Pills' claim, says the annotation, that the pills flush the poisons out of the system with two pints of bile and that they are the standard medical formula for waking up the liver. Each pill, says the British Medical Journal, is said to contain $0.25 \mathrm{gr}$. of Curaç xo aloe and $0.0625 \mathrm{gr}$. of Podophylli resin. The annotation adds: "These experiments point to a distant goal-the authoritative study of the pharmacological action of all proprietary preparations and the unbiased publications of the results".

That the Newspaper Proprietors' Association is alive to the importance of control of the advertisement of proprietary medicines is shown by the following rules governing such advertisements which have been unanimously adopted at a recent meeting of the Association. They appear in the British Medical Journal of October 9, p. 462.

(1) No advertisement will be accepted by the newspapers represented in the Newspaper Proprietors' Association which offers for sale to the public any medicine or treatment which is directly or by implication held out in terms calculated to le d to the belief that the medicine is effective in : (a) The treatment of Bright's disease, cancer, tuberculosis or consumption, diabetes, epilepsy, fits, locomotor ataxy, cataract, glaucoma, disseminated sclerosis, osteoarthritis, spinal, cerebral and venereal diseases, lupus, or paralysis or for preventing any of those ailments. (b) For the cure of amenorrhoea, hernia, blindness, rheumatoid arthritis, or any structural or organic ailment of the auditory system. (c) For procuring the miscarriage of women. (d) For the treatment of habits associated with sexual indulgence or for any ailment associated with those haliis.

(2) No advertisement will le accepted from an advertiser who by printed matter, orally, or in his advertisement undertakes: $(a)$ To diagnose by correspondence diseased conditions or any particular diseased conditions in a human leing or to receive from any person a statement of his or any other person's symptoms of ill-health with the view of advising as to, or providing for, the treatment of such ill-health by correspondence; or $(b)$, to treat by correspondence any of the ailments specified in Section I above.

(3) No advertisement will be inserted containing a testimonial other than one limited to the actual views of the writer, or any testimonial given by a doctor other than a recognized, sritish medical practitioner unless it is manifest that the doctor is not a British doctor of medicine.

(4) No advertisement will te accepted containing illustrations which are distorted or exiggerated in such a manner as to convey false impressions.

(5) No advertisement will ke accepted which in any way may lead persons to belier e that the product recommended emanates from any ho pital or oiticial source or is other than a proprietary medicine adver. tised by a particular manufacturer for the purpose specified, unless the advertising agent sulmitting the copy declares that the authority of such hospital or official source.had been duly obtained.

These rules are now in operation in all the London morning, evening and Sunday newspapers and all advertisements will, in addition to conforming to these rules, be submitted to medical scrutiny and the product to chemical analysis if it is considered necessary. This is the first time that the national newspapers of Great Britain have unanimously laid down and insisted on a standard of control over statements and claims made in advertisements.

\section{A SYNTHESIS OF A 42- CHROMOSOME WHEAT}

THE solution of the jigsaw-like puzzle of the origin of the 42-chromosome wheats of the 'vulgare' or bread-wheat type has been attempted by various methods. A recent endeavour to synthesize a bread-wheat by crossing Triticum turgidum $(2 n=28)$ with Aegilops speltoides $(2 n=14)$ adds a new piece to the puzzle (W. P. Thompson, E. J. Britten and Jean C. Harding, Canad. J. Res., C, 21, 134; 1943). It is generally agreed that the monococcum group of wheats $(2 n=14)$ has the chromosome sets $A A$ and the majority of the emmers $(2 n=28)$ the genoms $A A$ and $B B$, while all the bread-wheats have the constitution $A A B B C C$, the $C$ sets being derived in some way from some species of the genus Aegilops, one of the specios of which, Aegilops cylindrica, seems to have the genoms $C C D D$. The new species is the result of an attempt to repeat the hypothetical events in the origin of common wheat by first crossing one of the emmer section with a species of Aegilops with 14 chromosomes, and then producing the fertile amphidiploid by doubling the number of chromosomes with the aid of colchicine.

The new type has leaves with a single line of long hairs on the summit of each ridge, a hollow stem, the collar at the base of the spike open, rounded U. shaped glumes with wide truncated 'shoulders' and weakly developed keels, all of which are regarded as typical bread-wheat characters. In the bearded varieties of the vulgares, the awns are shorter than those of the emmers and in this character too the amphidiploid is near the bread-wheats. The teeth at the apex of the glumes are short and blunt as in most varieties of $T$. vulgare. The hairs of the brush at the apex of the grain are intermediate in number and length between the emmers and the vulgares. The rachis, however, is fragile, with a disarticulation which is characteristic of the emmers, and the slender rachis segments have their tops wider than the bases, as in the emmers.

In undoubled $F_{1}$ plants, the pollen mother-cells showed 1-3 trivalents in more than 80 per cent of the cells, while 5 or 6 bivalents occurred in 26 out of 57 cells, and 4-7 bivalents in 51 out of 57 cells. In the pollen mother cells of the amphidiploid plants the greater majority showed a few unpaired chromosomes as well as associations of three or four. $75-95$ per cent of the pollen appeared good and about three to a dozen grains were set in most heads. Backcrossing with $T$. vulgare and $T$. Spelta was possible both with the amphidiploid as the male or the female parent, but the resulting grains were badly shrivelled and germinated poorly. Of the 8 backcross hybrid plants obtained, 5 had 42 chromosomes and the remaining three 41,40 and 39 .

Thus it can well be said that a wheat-like species. which in most essentials is a vulgare type has been synthesized: a new piece has been found for the 